\title{
Gilles Laferté - L'embourgeoisement : une enquête chez les céréaliers
}

\section{Thierry Pouch}

\section{(2) OpenEdition}

Journals

Édition électronique

URL : https://journals.openedition.org/economierurale/9014

DOI : 10.4000/economierurale.9014

ISSN : 2105-2581

Éditeur

Société Française d'Économie Rurale (SFER)

Édition imprimée

Date de publication : 30 juin 2021

Pagination : 115-118

ISSN : 0013-0559

Référence électronique

Thierry Pouch, «Gilles Laferté - L'embourgeoisement : une enquête chez les céréaliers », Économie rurale [En ligne], 376 | Avril-juin, mis en ligne le 30 juin 2021, consulté le 06 janvier 2023. URL : http:// journals.openedition.org/economierurale/9014; DOI : https://doi.org/10.4000/economierurale.9014

Creative Commons - Attribution - Pas d'Utilisation Commerciale 4.0 International - CC BY-NC 4.0 https://creativecommons.org/licenses/by-nc/4.0/ 
Gilles LAFERTÉ

\section{L'embourgeoisement : une enquête chez les céréaliers}

Paris : Raisons d'agir, coll. « Cours et travaux », 2018, $365 \mathrm{p}$.

I ne se passe pas une semaine sans que 'on n'évoque, dans la sphère agricole et par voie de presse, les crises agricoles, la détresse des agriculteurs, leurs difficultés financières, quand ce n'est pas les drames humains engendrés par les cas de suicides dans une profession manifestement malmenée par les réformes de la Politique agricole commune (PAC) et par la mondialisation. À cet égard, l'ouvrage qu'a récemment publié Gilles Laferté, sociologue et chercheur à Dijon, pourrait-il le dit d'ailleurs lui-même dès les premières lignes de son introduction - passer pour une "provocation " et, s'il tombait entre les mains de responsables syndicaux ou d'agriculteurs de terrain, comme une sorte de trahison, émanant d'un chercheur de I'INRAE, institution ayant été depuis sa création un vecteur du développement agricole en France. L'auteur a toutefois l'intelligence d'indiquer à son lecteur que l'enquête qui a été réalisée s'est étalée sur plusieurs années, s'affranchissant ainsi du temps immédiat qui est celui de la presse et des médias. Les mutations, les bifurcations, les ruptures sont manifestement plus palpables sur un temps long pour le chercheur, dont c'est la mission, que sur celui, plus court, des journalistes. Ce qui est en jeu dans cette recherche, ce sont la mesure et les modalités de la mobilité sociale ascendante dont ont bénéficié les producteurs français de céréales, du moins ceux situés géographiquement dans l'Est de la France. Par ailleurs, c'est aussi la fonction même de la sociologie que de mettre au jour les choses sociales cachées, inavouées, qu'elles fassent ou non plaisir. C'est ce à quoi parvient l'auteur, qui, tant dans sa méthode que dans son analyse, puise une bonne part de son inspiration dans la sociologie de Pierre Bourdieu.

L'épais et passionnant ouvrage produit par Gilles Laferté se compose de deux parties, dans lesquelles se répartissent de façon équilibrée quatre chapitres. II restitue dans une analyse pénétrante un long travail d'enquête effectué dans le Germanois, aux confins de la Bourgogne et de la Champagne.

La première partie traite du processus d'embourgeoisement des céréaliers dans les rapports de production. La seconde des répercussions de cet embourgeoisement sur le mode de vie des céréaliers, leur rapport à l'école, à I'habitat, aux loisirs et à la ville. Ce processus illustre l'entrée dans la modernité d'une classe paysanne qui, jusque-là, posait au chercheur de redoutables problèmes de saisie et d'interprétation de cette classe, depuis au moins la seconde moitié du $\mathrm{XIX}$ siècle. L'embourgeoisement a essentiellement concerné les céréaliers, grands bénéficiaires de la politique agricole européenne, et de la modernisation des structures productives impulsées par les lois d'orientation de 1960-1962.

Une telle évolution a accentué d'une certaine manière la dynamique de la " dépaysannisation " de la France, parallèlement à la baisse du nombre des agriculteurs. Selon l'auteur, nous avons ainsi pu assister à une ascension sociale qui ne doit rien aux parcours scolaires des acteurs ou à leurs trajectoires culturelles, et davantage à la réussite économique. Les crises et le retrait de l'État de l'activité agricole, qui, depuis plus de vingt ans, ont occasionné la liquidation d'une frange des céréaliers, participent pourtant de cette dynamique d'embourgeoisement de ceux qui restent, à la faveur de l'agrandissement des exploitations et de leur ouverture à des modes de financement nouveaux.

Ce que dépeint dans cette recherche Gilles Laferté s'inscrit dans une problématique ancienne, celle de la survie, de 
la préservation de l'exploitation agricole familiale, dont le déploiement et la continuité ont longtemps caractérisé l'agriculture française mais qui, dans le capitalisme mondialisé, connaît une réelle érosion, laquelle suggère de rouvrir les analyses de Marx et de ses continuateurs sur l'absorption de l'agriculture par et dans le mode de production capitaliste. C'est tout l'intérêt de ce livre que de montrer en quoi l'embourgeoisement des céréaliers ne peut être interprété comme un processus individuel, mais bien comme la résultante de mutations " institutionnelles et marchandes macrosociales hors de portée des agriculteurs en question ${ }^{1}$, ayant touché un collectif de céréaliers.

L'une des manifestations concrètes de cet embourgeoisement a trait à la formation du revenu des céréaliers, qui d'après les données dont Gilles Laferté fait un usage approprié, est supérieur à celui des professions intermédiaires, juste en deçà de celui des artisans et commerçants, mais encore inférieur à celui des cadres. Fin connaisseur de son thème de recherche, l'auteur n'en rappelle pas moins que ce rattrapage monétaire demeure exposé aux fluctuations des prix de marché et aux réformes de la PAC, dont nous savons que I'un des fils conducteurs est précisément de diminuer les soutiens, lesquels avaient été l'un des fondements du processus d'embourgeoisement ${ }^{2}$. Abordant le thème des revenus des céréaliers, l'auteur approfondit son analyse en scrutant les points de tensions engendrés par l'élévation des revenus et donc du niveau de vie des producteurs de céréales (culpabilité vis-à-vis des valeurs fondatrices de l'agriculture familiale dans la République française, décalage envers l'idéologie méritocratique propre à la France, enrichissement fondé sur des soutiens publics en principe orientés vers les populations les plus en difficulté). Les pages 175-180 apparaissent, de ce point de vue, riches d'enseignements, puisqu'elles offrent aux lecteurs la possibilité de prendre la mesure des contradictions sociales mais aussi psychologiques dans lesquelles se situe une frange des agriculteurs français.

Parmi les nombreuses conséquences de cet embourgeoisement que Gilles Laferté passe en revue, nous trouvons d'abord celle du rapport au travail. L'ascension sociale des céréaliers se distingue par une réorientation du travail qu'ils effectuent, davantage en faveur de tâches managériales ou techniciennes et au détriment de celles d'exécution. Cela est d'autant plus prégnant que les structures se sont agrandies, entraînant parfois un renoncement à l'activité d'élevage laitier ${ }^{3}$. Une autre conséquence a trait au rapport à l'éducation, les céréaliers poussant leurs enfants à emprunter la voie des grandes études, au risque d'entraver le processus de transmission-installation, déjà bien abîmé. Le rapport à l'habitat, aux loisirs, à l'urbain, voire à la tenue vestimentaire, sont également analysés dans cette enquête, tout au long de la seconde partie.

Nous aurons compris que Gilles Laferté a pris un risque, celui de rompre avec une certaine vision "misérabiliste " de l'agriculture, qui, dans certains cas, reste fondée, afin de mettre au jour une vision plus en phase avec les aspirations sociales de certains agriculteurs. Ces aspirations-être des entrepreneurs par exemple - sont porteuses de contradictions dans lesquelles évoluent désormais les agriculteurs qui se sont embourgeoisés, qu'elles soient individuelles ou collectives. La démonstration avancée par l'auteur de cette recherche induit des interrogations profondes sur la mutation en cours du métier d'agriculteur, sur leur insertion dans le mode de production capitaliste, sur leur engagement dans le militantisme syndical, sur la reproduction économique et sociale de leur

1. Page 358.

2. Chapitre 4 de la partie I.

3. Le chapitre 3 de la partie I est fort important. 
profession, vue notamment sous I'angle de l'exploitation familiale.

Ce que décrit Gilles Laferté s'inscrit dans la sociologie de Pierre Bourdieu, qui avait qualifié, dans le cadre de ses recherches d'antan menées avec Abdelmalek Sayad sur la paysannerie en Algérie, la dynamique sociale du monde agricole de "dépaysannisation ». Dit autrement, le processus en cours illustre une forme de dislocation du groupe social formé par les agriculteurs, entraînant de ce fait une décomposition de leur cohésion politique et sociale. L'auteur suggère donc de ne pas se limiter à une vue quantitative de la crise que traverse le secteur agricole. De ce point de vue, la conclusion générale de l'ouvrage est magistrale. N'y aurait-il pas eu du coup ici l'opportunité de se demander si la " dépaysannisation » n'entrait pas en résonance avec le concept de "décomposition de la paysannerie " - ou du moins d'une certaine paysannerie - fort usité autrefois par des économistes ruraux se réclamant de Marx et de Lénine ?

Depuis plus de quarante ans, la diminution des effectifs d'agriculteurs, perceptible au gré des Recensements agricoles décennaux, n'est que la face apparente du malaise agricole. De même, la dérégulation des marchés agricoles, enclenchée avec la réforme de la PAC de 1992 et qui se caractérise en particulier par la baisse des soutiens publics, pour décisive qu'elle soit, mérite d'être complétée par une incursion dans la sociologie des agriculteurs. La mondialisation a pour sa part œuvré à cette dislocation du monde agricole, dans la mesure où, pour une fraction des agriculteurs, elle peut être appréhendée certes avec crainte, mais aussi comme une opportunité pour consolider leur appartenance à la sphère de l'entreprise. La mobilité sociale ascendante, ses conséquences sur l'identité des agriculteurs, aboutissent à de lourdes contraintes sur l'attractivité des institutions qui ont, historiquement, forgé cette identité (syndicalisme en mal de recrutement, organismes mutualistes...).
Des tensions en découlent, en particulier celle relative au désir de capter des "opportunités sociales " venues de l'extérieur, qui oblige à renoncer à la vertu intégratrice de la profession d'agriculteur ${ }^{4}$. C'est pourquoi cette mobilité ascendante, cet embourgeoisement des céréaliers, est source de conflits tant sociaux que personnels. La retranscription des entretiens apparaît de ce point de vue parlante, riche d'enseignements pour qui souhaite comprendre les ressorts du malaise actuel. Lisant entre les lignes, le lecteur peut alors retomber sur ce que disait Pierre Bourdieu de la psychanalyse, véritable cas particulier de la sociologie.

II manque toutefois au propos tenu par Gilles Laferté une dimension qui, faute de mieux, pourrait être qualifiée de prospective. Puisque ce sont des dispositifs institutionnels qui ont œuvré à la conversion sociale des céréaliers, à leur normalisation dans le capitalisme, et in fine à leur embourgeoisement - la dislocation de ces dispositifs, politiquement et socialement créés dans I'Union européenne, pose la question de la durabilité de cette mobilité ascendante. N'étant plus une priorité de l'Union européenne, l'agriculture est donc moins soutenue et est exposée aux pressions de la concurrence mondiale aiguisée par l'instauration d'Accords de libre-échange, ainsi qu'aux appétits économiques de nouveaux acteurs (financiers, puissances étrangères...), bref à un capitalisme dont la puissance d'expansion ne comporte pas de limites prédéfinies. Sans doute est-ce là que se niche la source du malaise actuel des agriculteurs, en l'occurrence des céréaliers, qui y voient la remise en cause, l'annonce de la fin imminente, d'un modèle qui leur a ouvert le chemin de leur conversion sociale. La peur du déclassement des céréaliers rejoint alors celle de la classe moyenne française dans son ensemble, les exposant ainsi à la violence du monde social.

4. Page 363. 
Au terme de cette recension sur un ouvrage de quelque 400 pages qui, dans le champ de la sociologie rurale, fera immanquablement date - du moins faut-il l'espérer et même le souhaiter -, ajoutons que l'auteur invite les chercheurs à sortir de leurs seuls périmètres scientifiques. À plusieurs reprises, il souligne que le malaise paysan, les crises successives, n'ont pas pour unique fondement les prix, le revenu ou les politiques publiques. Les ressorts de cette crise sont aussi sociologiques. Manière de penser que, dans la complexité du monde actuel, la pluridisciplinarité a un bel avenir et est un impératif. La sphère agricole demeure un formidable objet de recherche. L'ouvrage de Laferté en constitue une illustration supplémentaire.

Thierry POUCH APCA-DEAT-SERP Laboratoire REGARDS Université de Reims Champagne Ardenne 